\title{
PRAME Positive
}

National Cancer Institute

\section{Source}

National Cancer Institute. PRAME Positive. NCI Thesaurus. Code C133814.

An indication that PRAME expression has been detected in a sample. 